\title{
Surface core-level shifts for simple metals
}

\author{
Aldén, Magnus; Skriver, Hans Lomholt; Johansson, Börje
}

Published in:

Physical Review B

Link to article, DOI:

10.1103/PhysRevB.50.12118

Publication date:

1994

Document Version

Publisher's PDF, also known as Version of record

Link back to DTU Orbit

Citation (APA):

Aldén, M., Skriver, H. L., \& Johansson, B. (1994). Surface core-level shifts for simple metals. Physical Review B, 50(16), 12118-12130. https://doi.org/10.1103/PhysRevB.50.12118

\section{General rights}

Copyright and moral rights for the publications made accessible in the public portal are retained by the authors and/or other copyright owners and it is a condition of accessing publications that users recognise and abide by the legal requirements associated with these rights.

- Users may download and print one copy of any publication from the public portal for the purpose of private study or research.

- You may not further distribute the material or use it for any profit-making activity or commercial gain

- You may freely distribute the URL identifying the publication in the public portal

If you believe that this document breaches copyright please contact us providing details, and we will remove access to the work immediately and investigate your claim 


\title{
Surface core-level shifts for simple metals
}

\author{
Magnus Aldén \\ Condensed Matter Theory Group, Physics Department, Uppsala University, S-75121 Uppsala, Sweden \\ Hans L. Skriver \\ Center for Atomic-scale Materials Physics and Physics Department, \\ Technical University of Denmark, DK-2800 Lyngby, Denmark \\ Börje Johansson \\ Condensed Matter Theory Group, Physics Department, Uppsala University, S-75121 Uppsala, Sweden
}

(Received 27 May 1994)

\begin{abstract}
We have performed an $a b$ initio study of the surface core-level binding energy shift (SCLS) for 11 of the simple metals by means of a Green's-function technique within the tight-binding linearmuffin-tin-orbitals method. Initial- and final-state effects are included within the concept of complete screening, whereby a SCLS becomes equivalent to the surface segregation energy of a core-ionized atom, a quantity we obtain by separate bulk and surface impurity calculations. The results are in good agreement with experiment in most of those cases where the data originates from single-crystal measurements. We discuss the surface shifts of the electrostatic potentials and the band-centers in order to trace the microscopic origin of the SCLS in the simple metals and find that the anomalous subsurface core-level shifts in beryllium are caused by charge dipoles, which persist several layers into the bulk. We furthermore conclude that the unexpected negative sign of the SCLS in beryllium is predominantly an initial-state effect and is caused by the high electron density in this metal.
\end{abstract}

\section{INTRODUCTION}

Until recent years, the so-called simple, $s p$-bonded metals have to a lesser extent than the $5 d$ and $4 f$ transition metals been subjected to comprehensive studies of surface core-level binding energy shifts (SCLS). During the last one and a half decades the poineering SCLS measurements for gold ${ }^{1}$ and samarium ${ }^{2}$ have been followed by a considerable amount of work on notably the $5 d$ transition metal series where the comparatively sharp $4 f$ level is experimentally favorable to study with high precision. ${ }^{3,4}$ Hence the behavior of the SCLS across a transition metal series is generally well understood, ${ }^{3,5}$ although the importance of so-called inital- and final-state effects has been a controversial issue. ${ }^{6-11}$ In particular, due to the localized nature of the valence $d$ electrons in transition metals as compared to the $s p$ electrons, it has been possible to associate a so-called initial-state contribution to the SCLS with a model based on surface $d$-band narrowing and $d$-charge conservation at the surface. ${ }^{6,7,12}$

In the renewed interest shown by Wertheim and coworkers ${ }^{13-16}$ for surface core-level shifts in simple metals it was, for instance, pointed out by Riffe et al. ${ }^{15}$ that the band-narrowing model is not applicable to the alkali metals because they have free-electron-like bands, and that there exist no conclusive signs of $s p$ band narrowing at their surfaces. Still, the fact remains that the SCLS of the simple metals are in general positive ${ }^{4,15}$ as they would have been in analogy with early transition metals if this model could be applied. Apart from the more general tools provided by the so-called thermodynami- cal model, ${ }^{17,18}$ the comprehensive achievements reported from the theoretical side concerning these elements are, unexpectedly as it may seem, highly limited. The measurements performed by the mentioned authors and other workers $^{19-26}$ have nevertheless improved the experimental data base of simple metal SCLS to a level comparable to that of the $5 d$ transition metals, stressing the need for theoretical work.

The experimental efforts recently resulted in a layerresolved SCLS recording for $\mathrm{Be}(0001)$ by Johansson et al. ${ }^{27}$ where the data were presented using explicit support from $a b$ initio calculations. ${ }^{28}$ The beryllium shifts were found to exhibit a most unusual behavior in comparison with, on the one hand, the other simple metals, and on the other hand, most elemental metals in the Periodic Table. This is because, first, the measured and calculated SCLS for beryllium penetrate several layers deep down into the metal with an anomalously slow decrease in the magnitude of the shift. Second, and also observed in earlier work by Nyholm et al. ${ }^{22}$ the shift is negative in sharp contrast to the positive shifts usually observed for the simple metals. Third, the shift for the surface layer was found to be extremely large $(-0.82 \mathrm{eV})$. Hence, in these respects, beryllium seems to represent a unique physical situation. ${ }^{29}$

Here we present a theoretical study of the SCLS in 11 simple metals. We use a Green's-function technique within the tight-binding linear-muffin-tin-orbitals method $^{30}$ which earlier has been employed in extensive studies of surface energies and work functions, ${ }^{31,32}$ surface and interface magnetism, ${ }^{33-35}$ and stacking fault 
energies $^{36}$ of elemental metals. The present calculations are based on the impurity formulations ${ }^{37,11}$ of the Green's-function technique and therefore, as far as the Green's-function approach is concerned, partially related to the pseudopotential technique within a linear combination of atomic orbitals basis used by Feibelman ${ }^{38}$ in conjunction with a slab geometry and the $(Z+1)$ approximation for the core-ionized impurity. In the present work, however, we treat a surface as a completely semiinfinite entity and use the complete screening picture for the core-ionization process, i.e., we assume for metals that the symmetric part of the measured line profile of the core level corresponds to an electronically completely screened final state, in which the conduction electrons have attained a fully relaxed configuration in the presence of the core hole. As a result, the SCLS may be obtained as the surface segregation energy of the coreionized atom ${ }^{18,39,40}$ and the approach incorporates socalled initial-state as well as final-state effects.

\section{COMPUTATIONAL METHOD}

\section{A. The Green's-function techniques}

The tight-binding linear-muffin-tin-orbitals (TBLMTO) Green's-function technique as implemented for surfaces and interfaces by Skriver and Rosengaard ${ }^{30,31}$ is based on the generalized LMTO theory of Andersen and co-workers. ${ }^{37,41-44}$ The present Green's-function method differs from the slab approach by the fact that it takes proper account of the semi-infinite nature of a surface. This aspect relies on the ability, realized within the tightbinding representation and the atomic-sphere approximation (ASA), to generate the Green's-function matrices for a real, two-dimensional interface by an efficient procedure based on the principal layer technique ${ }^{45,46}$ which avoids $k$-point integration in the direction perpendicular to the surface. The technique allows us to carry the surface calculations to convergence in layer numbers which at least for the case of beryllium to be presented in Sec. IV appears to be difficult to do in a slab approach.

Within the complete screening picture, the SCLS may be obtained as the surface segregation energy of a neutral core-ionized atom, i.e., as the energy required to interchange an impurity atom in the bulk with a host atom at a surface site $Q_{s} \cdot{ }^{18,39,40}$ This energy may conveniently be expressed in terms of the computed impurity solution energies for the surface $\left(E_{\text {surf }}^{\text {sol }}\right)$ and bulk ( $\left.E_{\text {bulk }}^{\text {sol }}\right)$ as defined in Ref. 11. The SCLS is thereby obtained from

$$
\Delta_{c}=E_{\mathrm{surf}}^{\mathrm{sol}}-E_{\mathrm{bulk}}^{\mathrm{sol}}-E_{S, Q_{s}}
$$

where the last term represents the $Q_{s}$-projected surface energy of the host which within the ASA represents the energy required to transfer a host surface atom back into the bulk. ${ }^{47}$ The core-ionized atoms which enter the actual impurity calculations in Sec. III are taken from self-consistent atomic calculations where one electron has been transferred from the core level in question into the valence state. Hence, the description of the core-ionized atoms used here goes beyond the commonly employed $(Z+1)$ approximation.

The calculation of a segregation energy by means of (1) requires two separate impurity calculations, one with the impurity deep inside the bulk and one with the impurity at the surface. These separate calculations are in turn based on the original bulk impurity formulation by Gunnarsson et al. ${ }^{37}$ and the required Green's-functions for the perfect bulk crystal and the real surface are generated by means of the procedures described in Refs. 30 and 31, where for the surface calculations we use the principal layer technique. ${ }^{45,46}$ The Green's-functions for the impurity-perturbed crystals and surfaces are calculated self-consistently at the same level of accuracy in terms of, e.g., Hamiltonian, potentials, complex energy contour, and symmetrized Dyson equations ${ }^{11}$ and without any slab or supercell approximation. Thereby, the numerical errors in the calculated total-energy differences are reduced to a minimum.

\section{B. Corrected energy functional for the alkaline-earth metals}

An accurate theoretical determination of a surface core-level shift within the complete screening picture will of course rely on the quality of the calculated total energies involved in Eq. (1). In practice, one important factor which governs the accuracy of the calculated SCLS is the ability to account for the surface energy $E_{S}$, since the SCLS may be related to the surface energies by the simple estimate ${ }^{18,39}$

$$
\Delta_{c} \approx E_{S}^{\mathrm{Z}+1}-E_{S}^{Z}
$$

This expresses, within the $(Z+1)$ approximation and neglecting the impurity aspect, the surface core-level shift of the $Z$ metal as the difference between the surface energy of the $(Z+1)$ and $Z$ metals. It follows that an error in the calculated surface energy for either the $Z$ or the $(Z+1)$ metal will affect the accuracy of the shift, unless the errors are of the same size and cancel. ${ }^{5}$

Surface energies calculated by the present technique have been compared with experiment and full-potential slab calculations in Ref. 31 showing a high degree of accuracy for most transition metals. However, the surface energies of the the early transition metals Sc, Y, and La, which are the " $(Z+1)$-elements" of the alkalineearth metals $\mathrm{Ca}, \mathrm{Sr}$, and $\mathrm{Ba}$ are somewhat underestimated. This underestimate has been traced to a failure of the ASA energy functional and corrected for by including the nonspherically symmetric charge density in the calculation of the Coulomb and exchange-correlation contributions to the energy. ${ }^{48,49}$ The correction is most significant in the actinide series where the surface energy of $\mathrm{Th}$ and $\mathrm{Pa}$ otherwise would be negative. ${ }^{48}$ In the alkaline-earth part of the present study, where corrections to the ASA functional are needed, we shall use the simplified approach referred to in Ref. 49 as the spherical cell model (SCM), where the space integrations are performed over space-filling, overlapping atomic spheres 
TABLE I. Comparison between surface energies calculated within the ASA and SCM functionals as described in text.

\begin{tabular}{lcccccc}
\hline & & \multicolumn{2}{c}{ ASA } & \multicolumn{2}{c}{ SCM } & Expt. \\
Metal & Surface & $\mathrm{eV}$ & $\mathrm{J} / \mathrm{m}^{2}$ & $\mathrm{eV}$ & $\mathrm{J} / \mathrm{m}^{2}$ & $\mathrm{~J} / \mathrm{m}^{2}$ \\
\hline $\mathrm{Ca}$ & fcc (111) & 0.301 & 0.357 & 0.495 & 0.588 & 0.49 \\
$\mathrm{Sr}$ & fcc (111) & 0.290 & 0.290 & 0.448 & 0.448 & 0.41 \\
$\mathrm{Ba}$ & $\mathrm{bcc}(110)$ & 0.288 & 0.261 & 0.432 & 0.391 & 0.37 \\
\hline $\mathrm{Sc}$ & hcp (0001) & 0.494 & 0.836 & 1.010 & 1.707 & 1.28 \\
$\mathrm{Y}$ & hcp (0001) & 0.462 & 0.643 & 0.967 & 1.345 & 1.13 \\
Lu & hcp (0001) & 0.493 & 0.743 & 1.011 & 1.523 & 1.23 \\
\hline \hline
\end{tabular}

${ }^{a}$ de Boer et al. (surface independent) (Ref. 66).

rather than Wigner-Seitz cells. A comparison between surface energies calculated within the ASA as well as the SCM approach is given for relevant cases in Table I. The SCM energy functional will be used only for the alkaline-earth metals since in the other cases treated here the correction to the calculated SCLS will be small. For the alkali metals, for instance, the contribution to the SCLS from the nonspherically symmetric charge density is found to be $(\sim 0.02 \mathrm{eV})$ and, hence, may safely be neglected.

\section{Numerical details}

To perform bulk $k$-space integrations we use 1785 points in the irreducible wedge of the Brillouin zone for the bcc structure, 1505 points for the fcc structure, and 1500 points for the hcp structure. In the surface $k$ space integration we use 256 special points in the irreducible part of the two-dimensional Brillouin zone for the bcc(110) surface, 252 points for the fcc(111) and hcp $(0001)$ surfaces, and 136 points for the fcc(100) and bcc(100) surfaces. The Green's functions are sampled on a complex-energy contour, using 16 points distributed exponentially on a semicircle enclosing the occupied states. In the surface calculation, we use a surface region consisting of six layers of metal plus two layers of empty spheres simulating the vacuum. In the impurity calculations, we use cluster regions consisting of 51 atoms for the bcc crystal, 55 for the fcc crystal, and 51 for the hcp crystal. The impurity solution energies are thereby estimated to be converged to within a numerical error of less than $0.02 \mathrm{eV}$, utilizing the generalized phase shift formulation to sum the one-electron energies. ${ }^{37}$ We use an LMTO basis consisting of $s, p$, and $d$ orbitals, the frozen-core approximation, and include exchange and correlation in the parametrization of the local density approximation given by Vosko et al. ${ }^{50}$ We have applied a null test to our computational technique by performing full impurity calculations using a host atom to serve as the impurity and find that equalities $E_{\text {surf }}^{\text {sol }}=E_{S, Q_{s}}$ and $E_{\text {bulk }}^{\text {sol }}=0$ are obeyed to within a numerical accuracy of $0.01 \mathrm{eV}$ or better.

\section{CALCULATED SURFACE CORE-LEVEL SHIFTS FOR THE SIMPLE METALS}

In the following we shall present calculated surface core-level shifts for the monovalent alkali metals $(\mathrm{Li}, \mathrm{Na}$,
$\mathrm{K}, \mathrm{Rb}$, and $\mathrm{Cs}$ ), the divalent alkaline-earth metals (Be, $\mathrm{Mg}, \mathrm{Ca}, \mathrm{Sr}$, and $\mathrm{Ba}$ ), and trivalent aluminium ( $\mathrm{Al})$, as obtained by means of the procedure outlined in the preceding section and described in detail in Ref. 11. The core-level electron removed from the impurity atom is taken from the $1 s$ level in $\mathrm{Li}$ and $\mathrm{Be}$, the $2 p$ level in $\mathrm{Na}$, $\mathrm{Mg}$, and $\mathrm{Al}$, the $3 p$ level in $\mathrm{K}$ and $\mathrm{Ca}$, and the $4 p$ level in $\mathrm{Rb}$ and $\mathrm{Sr}$. In $\mathrm{Cs}$ we remove the core electron from the $4 d$ or the $5 p$ level since both of these are usually considered in experiments, whereas in $\mathrm{Ba}$ we remove only the $4 d$ level. The calculations are performed for the most close-packed surface of the experimentally observed bulk crystal structure and at the experimentally observed lattice spacings (and $c / a$ ratio for the hcp metals).

\section{A. The alkali metals}

Measurements of SCLS in the alkali metals have to our knowledge only been performed on polycrystalline films grown a few layers thick by deposition of alkali vapor on a metal substrate. Hence the SCLS refer to the corelevel binding energy shift between that of an atom at the surface and an atom in the center of the film, both separated from a possible signal from the interface atoms situated next to the substrate. ${ }^{26}$ One might therefore worry that the film-center reference level differs from that of the true bulk, that possible subsurface intensities invalidate a correct determination of the reference level, and that the possible existence of, e.g., a subsurface shift is never traced. As we shall see, however, the computed shift for the subsurface layer of the alkali metals is very smalltypically less than $30 \mathrm{meV}$ - suggesting that a separation of the subsurface signal is a difficult task even for a single-crystal face of a pure alkali metal, and that the assumption of a bulk-resemblant reference level is reasonable. Wertheim and co-workers claim to have grown, on a $\mathrm{Ni}(100)$ substrate, alkali layers with a well-ordered (110) surface orientation, ${ }^{16}$ i.e., presumably also in the bcc phase, whereas Lundgren et al. report less well characterized growth modes using an $\mathrm{Al}(111)$ substrate, and that the crystal structure of their $\mathrm{Na}$ film is fcc or hcp rather than bcc. ${ }^{26}$

The calculated SCLS for the bcc(110) surface of the alkali metals (Li, Na, K, Rb, and Cs) are listed in Table II and presented in Fig. 1 together with available recent experimental data. ${ }^{4,13,15,16,26}$ It is seen that for $\mathrm{Li}$ the experimental result of $0.52 \mathrm{eV}$ (Ref. 16) exceeds the theoretical value by approximately $0.1 \mathrm{eV}$, which is similar to the deviation between theory and experiment found in the neighboring element Be (Ref. 28) (see below). When proceeding from $\mathrm{Li}$ to the heavier elements $\mathrm{Na}$-Cs the experimental SCLS are reduced by more than a factor of two, and this reduction, which makes $\mathrm{Li}$ appear anomalous, is very well reproduced by the calculations. In fact, for $\mathrm{Na}-\mathrm{Cs}$ the agreement between the (110) calculations and experiment is of the order of the scatter in the measured data.

We have also calculated subsurface layer SCLS in order to provide a consistent data base to be compared with the anomalous sublayer shifts of the alkaline-earth 


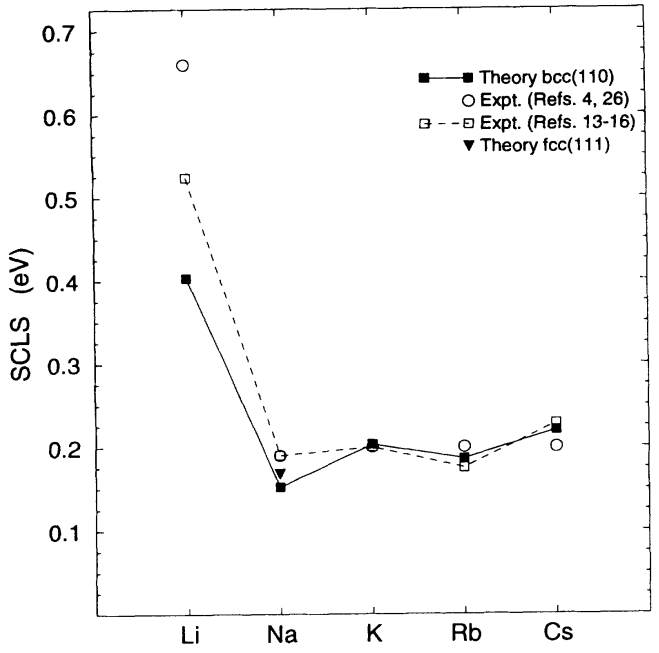

FIG. 1. Comparison between calculated and measured surface core-level shifts of the alkali metals. The calculations for the bcc(110) surface are marked with filled squares. For $\mathrm{Na}$ we have also included a calculation for the fcc(111) facet, denoted by a filled triangle. Experimental data are marked with open symbols, referring separately to (a) the studies by Wertheim and co-workers (Refs. 13-16) (open squares), and to (b) those of other workers (open circles) for Li (Ref. 4) and Na-Cs (Ref. 26).

metal beryllium. ${ }^{27,28}$ The results for the alkali metals are included in Table II, where it may be seen that the sublayer shifts are indeed small, also for $\mathrm{Li}$ which is next to $\mathrm{Be}$ in the Periodic Table. Hence, in terms of sublayer shifts $\mathrm{Li}$ behaves similarly to the other alkali elements.

In Fig. 2 we examine the $(Z+1)$ approximation by comparing the results for the bcc(110) face given in Fig. 1 and those obtained in calculations where the core-hole impurity in the $Z$ metal host has been replaced by the $(Z+1)$ atom. The difference is significant for $\mathrm{Li}$ but becomes quite small for $\mathrm{Na}$ and the heavier elements.

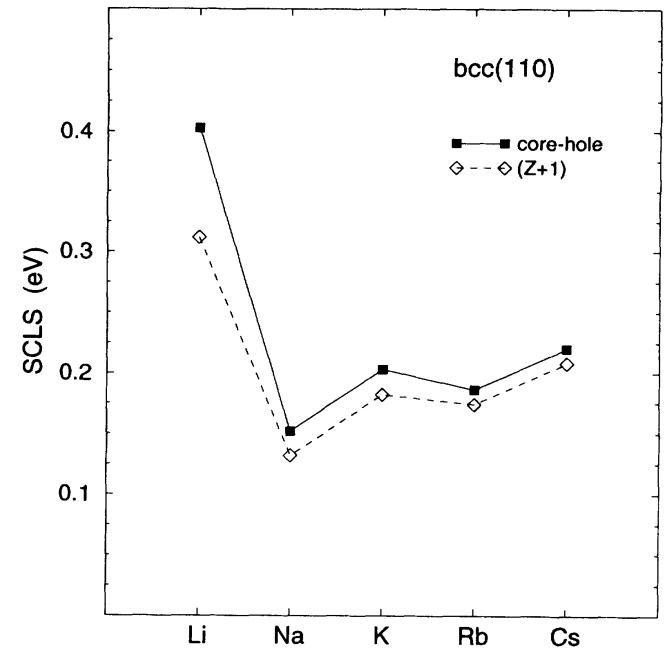

FIG. 2. Study of the $(Z+1)$ approximation for the alkali metals, by comparing calculations for the bcc(110) facet where the impurity is treated as (a) a neutral, core-ionized host atom as described in text (solid squares), and (b) a $(Z+1)$ atom in the $Z$ metal host (open diamonds).

Hence, there appears to be a correlation between the SCLS on the one hand and the relative effect of the missing core electron on the spatial distribution of the core on the other, and the effect seems to be reduced in the heavier elements in comparison with the lighter ones. For transition metals, the error of the $(Z+1)$ approximation was found to be at its largest in the elements in the middle of the series, ${ }^{5}$ i.e., in the elements in which the size of the core is large in comparison with the relatively compressed atomic volumes. ${ }^{51-53}$ However, it should be pointed out that because of the use of the frozen-core approximation in our calculations a complete assessment of the validity of the $(Z+1)$ approximation cannot be made.

In Fig. 3 we show the initial-state $s, p$, and $d$ occupancies, as obtained within LMTO theory and projected

TABLE II. First $(S)$, second $(S-1)$, and third $(S-2)$ surface core-level shifts of the alkali metals.

\begin{tabular}{lccccc}
\hline \hline & & & \multicolumn{3}{c}{ SCLS (eV) } \\
Metal & Surface & $S-2$ & $S-1$ & $S$ & Expt. $(S)$ \\
\hline $\operatorname{Li}(1 s)$ & bcc (110) & 0.008 & -0.008 & 0.403 & $0.524,,^{\mathrm{a}} 0.66^{\mathrm{b}}$ \\
& bcc (100) & -0.065 & 0.039 & 0.698 & \\
\hline $\mathrm{Na}(2 p)$ & bcc (110) & -0.005 & -0.027 & 0.152 & $0.184-0.195^{\mathrm{a}}$ \\
& fcc (111) & & -0.028 & 0.168 & $0.19^{\mathrm{c}}$ \\
& bcc (100) & -0.029 & -0.022 & 0.268 & \\
\hline $\mathrm{K}(3 p)$ & bcc (110) & 0.004 & -0.025 & 0.203 & $0.20,{ }^{\mathrm{d}} 0.20^{\mathrm{c}}$ \\
& bcc (100) & 0.019 & 0.001 & 0.302 & \\
\hline $\mathrm{Rb}(4 p)$ & bcc (110) & 0.008 & -0.022 & 0.186 & $0.175,{ }^{\mathrm{a}} 0.19,{ }^{\mathrm{d}} 0.20^{\mathrm{c}}$ \\
& bcc (100) & 0.026 & -0.001 & 0.266 & \\
\hline $\mathrm{Cs}(4 d)$ & bcc (110) & 0.015 & 0.005 & 0.220 & $0.20^{\mathrm{c}}$ \\
$\mathrm{Cs}(5 p)$ & bcc (110) & 0.016 & -0.010 & 0.218 & $0.228,{ }^{\mathrm{e}} 0.22^{\mathrm{c}}$ \\
$\mathrm{Cs}(4 d)$ & bcc (100) & 0.052 & 0.022 & 0.322 & \\
\hline \hline
\end{tabular}

${ }^{a}$ Wertheim et al. (Ref. 16).

${ }^{b}$ Flodström et al. (Ref. 4).

${ }^{c}$ Lundgren et al. (Ref. 26).

${ }^{\mathrm{d}}$ Riffe et al. (Ref. 15).

${ }^{\mathrm{e}}$ Wertheim et al. (Ref. 13). 


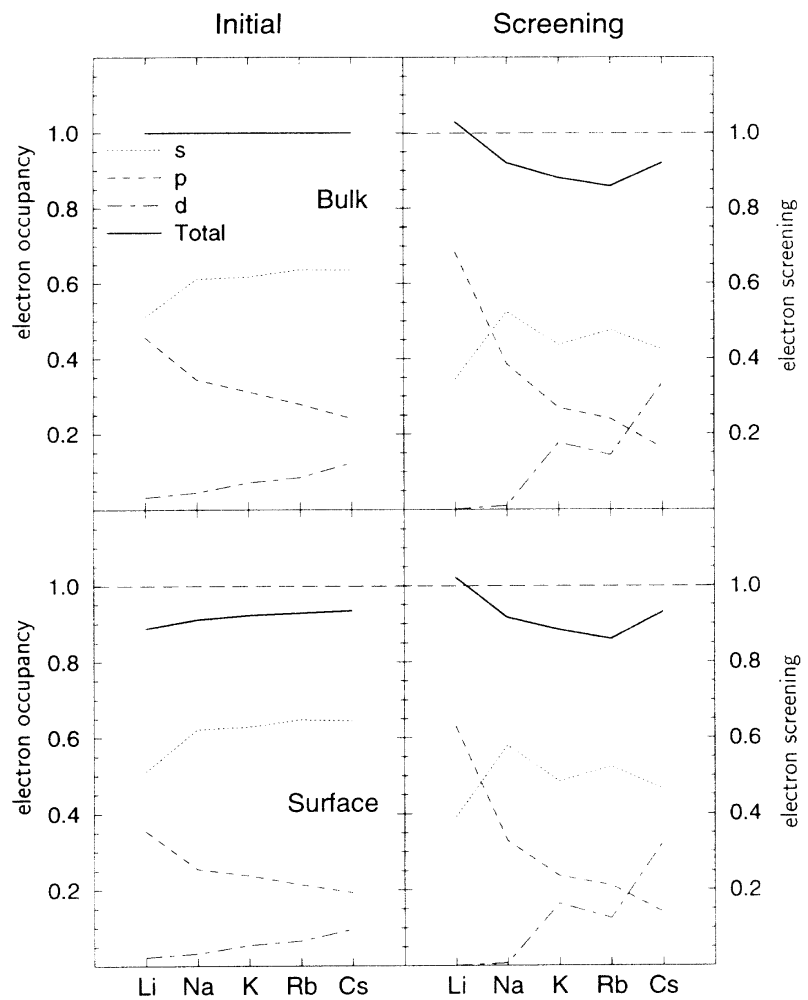

FIG. 3. Left: calculated initial-state valence electron occupancies in the bulk (top) and at the surface layer (bottom) of the alkali metals, given in terms of the partial $s, p$, and $d$ occupations. Right: screening charge (see text) on the core-ionized impurity in the bulk (top) and at the surface (bottom), projected on the partial $s, p$, and $d$ occupations.

onto the bulk and surface atomic spheres, respectively, before core ionization. In addition, we present in the figure the screening charge, defined as the number of electrons on the impurity site after the core ionization minus the number of electrons on the site before the ionization. This charge has also been divided into partial $s, p$, and $d$ occupations. For the screening charge, the main change down the alkali column is seen to occur in the $p$ and $d$ contributions. The former dominates in $\mathrm{Li}$ but is significantly reduced in $\mathrm{Cs}$, where instead the magnitude of the $d$ screening is comparable to that of the $s$ and $p$ states. The interchange of the amount of $p$ and $d$ type screening which occurs in the sequence $\mathrm{Na}$-Cs does not seem to affect the magnitude of the calculated SCLS. However, a closer inspection of Fig. 1 reveals that the calculated small SCLS variations between $\mathrm{Na}, \mathrm{K}, \mathrm{Rb}$, and Cs, also detected experimentally in Refs. 15 and 16, correlate with the computed $d$ screening (Fig. 3) and with the surface shift of one-electron $s p$ band centers (Sec. IV B) for these elements.

\section{B. The alkaline-earth metals}

In the case of the alkaline-earth metals the experimental SCLS information is more limited than it is for the alkali metals. For instance, no data have been reported for $\mathrm{Ca}$ and $\mathrm{Sr}$, and only a single observation is found in the literature for $\mathrm{Mg}$ (Ref. 21) and Ba (Ref. 23), respectively. The data for hcp $\mathrm{Mg}$ was taken from a (0001)oriented polished single crystal, ${ }^{54}$ giving a SCLS of 0.14 eV. ${ }^{21}$ The value for $\mathrm{Ba}, 0.48 \mathrm{eV}$, refers to measurements made on a polycrystalline $\mathrm{Ba}$ film prepared on an $\mathrm{Al}(111)$ substrate. ${ }^{23}$ Finally, for polycrystalline Be a negative surface core-level shift $(-0.50 \mathrm{eV})$ was first reported by $\mathrm{Ny}$ holm et al. ${ }^{22}$ later verified by layer-resolved recordings for single-crystal $\mathrm{Be}(0001),{ }^{27}$ where a shift of $-0.82 \mathrm{eV}$ was interpreted to originate from the surface layer, -0.57 $\mathrm{eV}$ from the first subsurface layer, and $\mathrm{a}-0.26 \mathrm{eV}$ shift from the second subsurface layer.

The calculated SCLS for the most close-packed surface of the observed crystal structure for Be (hcp), Mg (hcp), $\mathrm{Ca}$ (fcc), $\mathrm{Sr}$ (fcc), and $\mathrm{Ba}$ (bcc) are presented in Fig. 4, together with available experimental data. In the figure, we have explicitly included the abnormal subsurface layer shifts in $\mathrm{Be}(0001)$ but only the shifts corresponding to the surface layer in $\mathrm{Mg}(0001), \mathrm{Ca}(111), \mathrm{Sr}(111)$, and $\mathrm{Ba}(110)$, since in these elements the sublayer shifts are very small (see Table III).

As discussed in Sec. II B the usage of the SCM functional is expected to improve the accuracy of the calculated SCLS of the alkaline earths. Therefore, we present in Fig. 4 theoretical values obtained by means of the the SCM functional but include also ASA values in order to make a connection to our previous ASA results for $\mathrm{Be}, \mathrm{Mg},{ }^{28}$ and $\mathrm{Sr}^{5,11}$ It may be seen in the figure that the SCM correction improves the agreement with experiment in all cases, e.g., also for $\mathrm{Be}$ and $\mathrm{Mg}$. However, the SCM correction is considerably smaller than may be es-

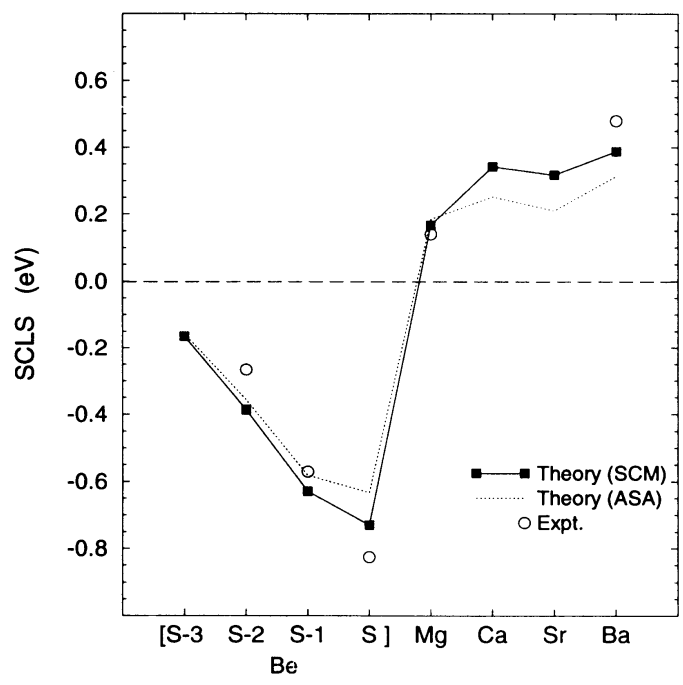

FIG. 4. Comparison between calculated and experimental SCLS for the alkaline-earth metals. The calculations are for the most close-packed surface, and refer separately to the SCM total-energy functional (solid squares) and the ASA functional (dotted line), as described in text. Experimental data are marked with open circles: the polycrystalline $\mathrm{Ba}$ (Ref. 23), and single-crystal Be (Ref. 27) and Mg (Ref. 21) measurements. ( $S-1$ denotes the first Be subsurface layer, $S-2$ the second Be subsurface layer, etc.) 
TABLE III. First $(S)$, second $(S-1)$, and third $(S-2)$ surface core-level shifts of the alkaline-earth metals calculated by means of the SCM functional. A comparison with the ASA functional is given for $\mathrm{Be}$ and $\mathrm{Mg}$.

\begin{tabular}{lccccc}
\hline \hline & & & \multicolumn{2}{c}{ SCLS (eV) } & Expt. $(S)$ \\
Metal & Surface & $S-2$ & $S-1$ & $S$ & $0.14^{\mathrm{c}}$ \\
$\mathrm{Be}(1 s)$ & hcp (0001) & -0.387 & -0.629 & -0.729 & {$[-0.265,-0.570,-0.825]^{\mathrm{a}}-0.50^{\mathrm{b}}$} \\
$\mathrm{Be}(1 s)$ ASA & hcp (0001) & -0.357 & -0.581 & -0.633 & \\
\hline $\mathrm{Mg}(2 p)$ & hcp (0001) & -0.022 & -0.001 & 0.167 & \\
$\mathrm{Mg}(2 p)$ ASA & hcp (0001) & -0.018 & 0.001 & 0.185 & $0.48^{\text {d }}$ \\
\hline $\mathrm{Ca}(3 p)$ & fcc (111) & 0.039 & 0.048 & 0.343 & \\
\hline $\mathrm{Sr}(4 p)$ & fcc (111) & 0.025 & 0.016 & 0.318 & \\
$\mathrm{Ba}(4 d)$ & bcc (110) & & 0.032 & 0.388 & \\
& bcc (100) & & -0.052 & 0.598 & \\
\hline \hline
\end{tabular}

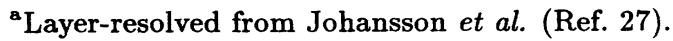

${ }^{\mathrm{b}}$ Nyholm et al. (Ref. 22).

${ }^{\mathrm{c}}$ Kammerer et al. (Ref. 21).

d Jacobi et al. (Ref. 23).

timated on the basis of the approximate relation (2) and the surface energies in Table I. This may be attributed to the fact that in deriving ${ }^{18}(2)$ one incorrectly invokes total rather than site-projected surface energies ${ }^{7,11}$ and neglects the impurity effects.

The experimental SCLS in Be shown in Fig. 4 are seen to be negative, exceptionally large, and to penetrate far into the surface region. All of these trends are well reproduced by the present theory, and the best agreement is found for the first subsurface layer $(S-1)$. The magnitude of the surface shift $(S)$ is seen to be underestimated by approximately $0.1 \mathrm{eV}$, which may partly be attributed to the neglect of layer relaxation in the present type of calculations. This neglect can be significant because the surface layer relaxation in $\mathrm{Be}(0001)$ is found to be unusually large and outwardly directed. ${ }^{55,56}$ In the second subsurface layer $(S-2)$ the experimental value lies midway between the theoretical $(S-2)$ and $(S-3)$ shifts, and hence we speculate that this discrepancy might be due to the experimental difficulty of resolving the $(S-2)$ and $(S-3)$ shifts, ${ }^{54}$ if not caused by other computational shortcomings.

In contrast to $\mathrm{Be}$ the later group IIA elements $\mathrm{Mg}$-Ba shown Fig. 4 have small positive SCLS in accordance with the behavior expected for simple metals. We find that the small $(0.14 \mathrm{eV})$ shift observed in $\mathrm{Mg}$ (Ref. 21$)$ is only slightly overestimated by the theoretical SCM result $(0.167 \mathrm{eV})$ obtained for $\mathrm{Mg}(0001)$ (Table II). Proceeding from $\mathrm{Mg}$ to $\mathrm{Ca}, \mathrm{Sr}$, and $\mathrm{Ba}$ the calculated SCLS are now almost twice as large as for the alkali metals which we may partly attribute to the change from $s p$-type screening in $\mathrm{Be}$ and $\mathrm{Mg}$ to $d$-type screening in $\mathrm{Ca}, \mathrm{Sr}$, and $\mathrm{Ba}$ as demonstrated in Fig. 5, and, perhaps more importantly, to the enhanced surface $s p$ band-center shifts exhibited by the divalent metals (Sec. IV B). In the case of $\mathrm{Ba}$ the computed shift is $0.1 \mathrm{eV}$ lower than the observed value, which is a rather small discrepancy in view of the fact that the measurements refer to a polycrystalline sample. ${ }^{23}$

The angular-momentum characteristics of the initialstate occupation and screening charge of the core-ionized atom presented in Fig. 5 reveals for Be a strong dominance of $p$ states. In the final state in bulk Be these amount to 2.0 electrons, which should be compared to only 1.5 for $\mathrm{Mg}$ (compensated by an increased $s$ character). The above mentioned connection between enhanced SCLS, $d$-screening, and the band-center shift, as obtained for the pretransition elements $\mathrm{Ca}-\mathrm{Ba}$, appears to be consistent with the detailed variation in the $d$-type screening (Fig. 5), the surface band-center (potential) shift (en-

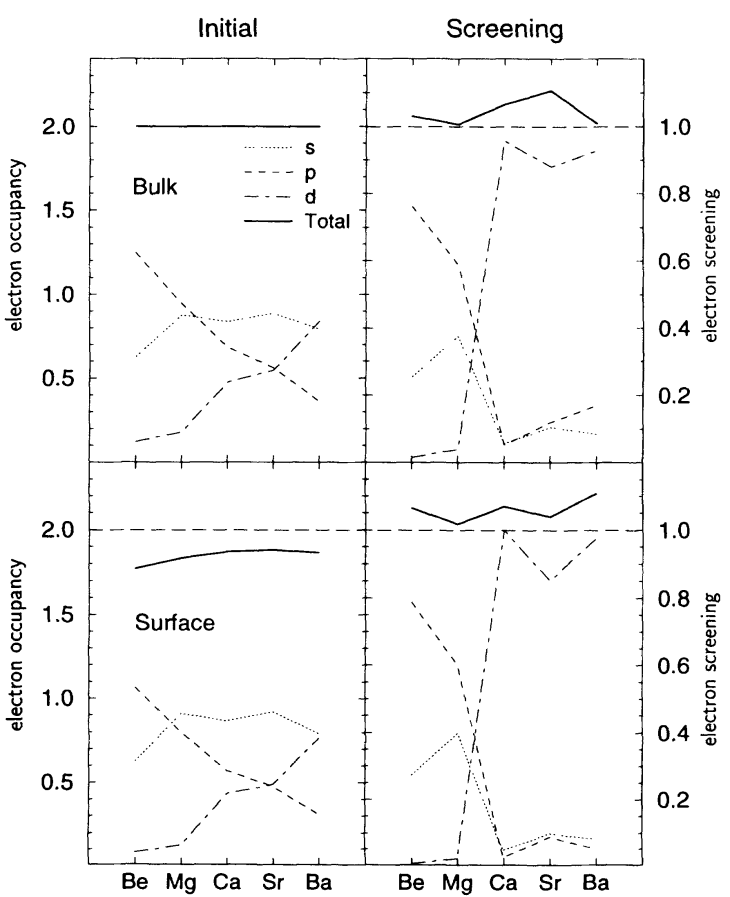

FIG. 5. Left: calculated initial-state valence electron occupancies in the bulk (top) and at the surface layer (bottom) of the alkaline-earth metals, given in terms of the partial $s, p$, and $d$ occupations. Right: screening charge on the core-ionized impurity in the bulk (top) and at the surface (bottom), projected on the partial $s, p$, and $d$ occupations. 
countered in Sec. IV), and SCLS (Fig. 4) for these elements, i.e., in complete analogy with the case of the alkali metals.

\section{Aluminium}

A comparatively large amount of work both experimental ${ }^{19-21,24,25}$ and theoretical ${ }^{57,38,24,58}$ has been devoted to the study of the trivalent metal Al. Here, the SCLS experiments have traditionally been performed on single crystals and at several surface orientations. In the case of the most frequently studied Al surface, fcc $(100)$, the measured SCLS data range from $-0.040 \mathrm{eV}$ to -0.120 $\mathrm{eV},{ }^{19-21,25}$ except for the single value of $+0.20 \mathrm{eV}$ reported by Bagus et al. ${ }^{24}$ In the most recent of the $\mathrm{Al}(100)$ experiments, Nyholm and co-workers found a separation of $-0.096 \mathrm{eV}$ between the surface and bulk signal. ${ }^{25}$ In the case of the fcc(111) face Nyholm et al. ${ }^{25}$ report an experimental shift close to zero with an upper limit of $+0.015 \mathrm{eV}$.

We have calculated the SCLS corresponding to the $2 p$ level in $\mathrm{Al}$ for two close-packed surfaces by means of the ASA functional and find small positive shifts. For the fcc(111) face we find $0.011 \mathrm{eV}$ in good agreement with the experimental shift, ${ }^{25}$ while for fcc(100) we find a shift of $0.004 \mathrm{eV}$ which is approximately $0.1 \mathrm{eV}$ higher than the experimental $^{25}$ as well as the theoretical value obtained by Feibelman. ${ }^{38}$ If, however, we use the $(Z+1)$ approximation, i.e., replace the $2 p$ hole impurity by a $\mathrm{Si}$ atom, as was indeed done by Feibelman, the Al(100) SCLS becomes $-0.110 \mathrm{eV}$, in almost perfect agreement with his result. Thus, the perfect agreement between previous theory and experiment may in part be a result of the $(Z+1)$ approximation.

\section{BONDING IN THE INITIAL AND FINAL STATES}

In the present study, we have provided theoretical SCLS data for the most close-packed surface of the $s p$ bonded simple metals by means of $a b$ initio total energy calculations as dictated by the complete screening picture of the core-ionization process. A SCLS is therefore obtained as the surface segregation energy of a neutral, core-ionized atom, ${ }^{18,39,40}$ involving separate Green's-function impurity calculations for the bulk and surface, respectively. The agreement between theory and experiment is in general very satisfactory, suggesting that the approximations on which the computational technique is based are well founded and that for the cases where experimental single-crystal data is still missing, notably in the alkaline-earth series, the calculations should provide reliable predictions. In addition, the observed agreement lends explicit support to the assumption of associating the main peak in the observed spectral features with an electronically fully relaxed core-hole state, and hence to the identification of the SCLS using de facto two different final states which the separate impurity calculations are tailored to describe.
However, there exists at present no satisfactory understanding of the behavior of the SCLS in the simple metals. The fact that the shifts are positive in most cases may, to some extent, be interpreted within the thermodynamical model as, for instance, formulated by the simple estimate (2). Unfortunately, such an approach gives very little insight into the microscopic origin of the SCLS. In particular, the thermodynamical model is not capable of explaining the anomalous subsurface shifts in beryllium since it does not probe local properties. Hence for the understanding of these shifts we shall first resort to a microscopic variable, namely, the local, layer-dependent one-electron potential.

\section{A. Surface potential}

The spill out of charge into the vacuum which occurs at a surface gives rise to a dipole barrier in the electrostatic potential. Hence, the work function $W$ may be expressed by the well-known relation

$$
W=\Delta \phi-\epsilon_{F},
$$

where $\epsilon_{F}$ is the Fermi level and $\Delta \phi$ the dipole barrier which may be obtained from the difference in the envelope function of the electrostatic potential ${ }^{30}$

$$
\Delta \phi=\bar{V}_{C}(R \rightarrow \text { vacuum })-\bar{V}_{C}(R \rightarrow \text { bulk })
$$

between that of the pure vacuum and bulk, respectively. This dipole barrier represents the accumulated potential rise when proceeding from the bulk to the vacuum, but gives no information on the local potential in each layer, which is needed if the SCLS are to be interpreted within the commonly used initial-state framework.

Within the ASA, as defined for surfaces in Ref. 30, the envelope function of the potential is determined by the electrostatic monopole $Q_{R}$ and dipole $P_{z ; R}$ in the atomic sphere at $R$ and the dipole barrier may be obtained as the sum

$$
\Delta \phi=\frac{1}{S}\left(\frac{8 \pi S^{2}}{A}\right) \sum_{R}\left(Q_{R} \frac{R_{z}}{S}+\sqrt{3} P_{z ; R}\right)
$$

over atomic positions in the surface region. In (5), $S$ is the average Wigner-Seitz radius, $A$ the area of the twodimensional unit cell, and $R_{z}$ the position of the atomic sphere projected onto the direction perpendicular to the surface. The layer-dependent envelope of the local potential, i.e. the one-electron potential at the surface of an atomic sphere, is given by the monopole and dipole parts of the multipole expansion

$$
\begin{aligned}
\bar{V}_{C}(R)= & {\left[\frac{2 Q_{R}}{S_{R}}+\frac{1}{S} \sum_{R^{\prime}} M_{R R^{\prime}}^{s s} Q_{R^{\prime}}\right] } \\
& +\frac{1}{S} \sum_{R^{\prime}} M_{R R^{\prime}}^{s z} P_{z ; R^{\prime}}
\end{aligned}
$$

where $M^{s s}$ and $M^{s z}$ are the multipole matrices for a two-dimensional interface. ${ }^{30}$ In (6) the term in brack- 
ets describes the potential due to the charge transfer (monopoles) corrected by the local atomic-sphere boundary condition $2 Q_{R} / S_{R}$, and the last term is the dipole potential from the dipoles in the perturbed layers in the bulk/vacuum surface region. As a result, the local potential envelope

$$
\bar{V}_{C}(R)=V_{Q}+V_{P_{z}}
$$

at a particular layer $R$ may be analyzed in terms of separate monopole $V_{Q}$ and dipole $V_{P_{z}}$ contributions.

\section{B. The anomalous surface core-revel shift in Be}

Calculated monopoles (charge transfer) and dipoles for the surfaces of $\mathrm{Li}, \mathrm{Be}$, and $\mathrm{Mg}$ are presented in Fig. 6 . The left panel shows the charge transfer $Q$ together with the induced dipole moments $P_{z}$ which represent the main deviation from charge homogenity in each sphere. It is seen that the charge transfer in Be is approximately twice that in $\mathrm{Li}$ and $\mathrm{Mg}$, but as far as the dipole behavior in the $(S)$ and $(S+1)$ layers is concerned there appears no essential difference between these elements. Moreover, on the scale used in the left panel and as expected for a metal all three elements exhibit similar fast convergence

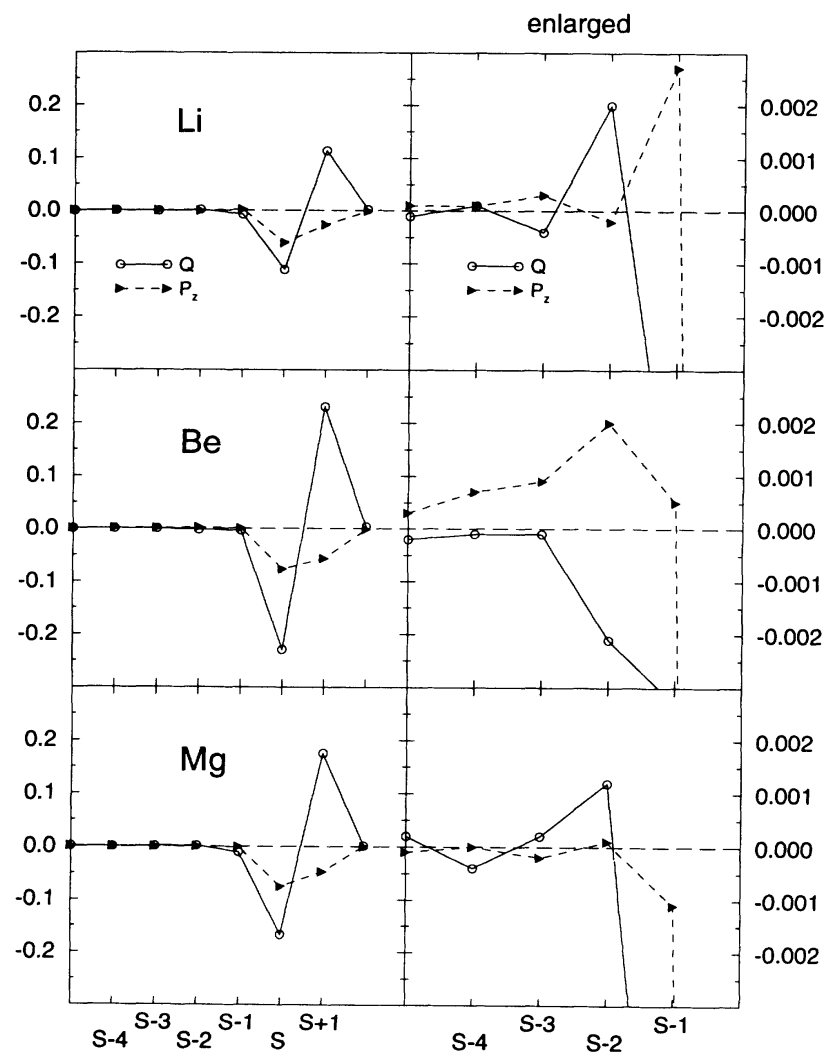

FIG. 6. Layer-projected charges $(Q)$ and dipoles $\left(P_{z}\right)$ in the surface region for $\mathrm{Li}, \mathrm{Be}$, and $\mathrm{Mg}$. The scale used on the left-hand side is enlarged to the right by a factor of exactly 100. Charges and dipoles are given in units of $-|e|$ and $-|e|($ a.u. length), respectively. ( $S$ denotes the surface layer, $S-1$ the first subsurface layer, etc.) in the electronic properties when one proceeds into the bulk.

However, on the scale used in the right-hand panel of Fig. 6 there appear qualitative as well as quantitative differences between $\mathrm{Li}, \mathrm{Be}$, and $\mathrm{Mg}$. In $\mathrm{Li}$ and $\mathrm{Mg}$, the charge density oscillates through the surface layers as typically obtained, although with a different magnitude, in jellium-type surface calculations, ${ }^{59,60}$ whereas this behavior is suppressed in Be. The dipole moment in $\mathrm{Li}$ and Mg oscillates similarly but with extremely small amplitudes. In contrast, the dipoles moment in $\mathrm{Be}$ is anomalously large and, in addition, decreases exceedingly slowly and monotonically from the $(S-2)$ layer through the next 3-4 layers towards the bulk region. There is therefore a significant net dipole in the Be subsurface region below the $(S-1)$ layer, and the corresponding dipole field from each layer is added constructively to the dipole field from the monopoles and this gives, as we shall see, a crucial contribution to the total subsurface potential.

In Fig. 7 we present the electrostatic potential envelope for $\mathrm{Li}, \mathrm{Be}$, and $\mathrm{Mg}$ as given by the monopole and dipole moments shown in Fig. 6 and separated according to Eqs. (6) and (7). The left-hand panel shows the cancellation which takes place between the monopole and dipole contributions and which forms a positive net surface dipole barrier. It is seen that this barrier which is the dominant contribution to the work function is significantly larger in $\mathrm{Be}$ than in $\mathrm{Li}$ and $\mathrm{Mg}$, and this is

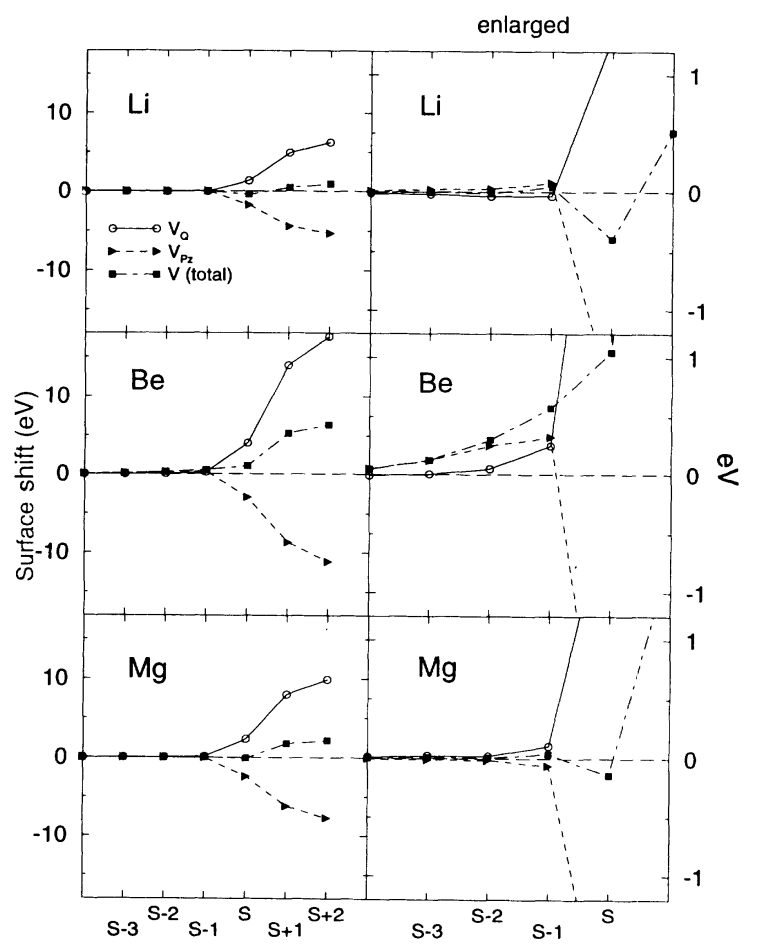

FIG. 7. Layer-projected surface shift of the envelope function of the local potential, as monitored in terms of monopole and dipole contributions. Left side shows the potential shifts on the scale of the dipole barrier contributions. This scale is enlarged on the right-hand side figures by a factor of about 15. ( $S$ denotes the surface layer, $S-1$ the first subsurface layer, etc.) 
of course in complete agreement with the fact that the work function computed by (3) to be $5.62 \mathrm{eV}$ for $\mathrm{Be}$ is much larger than the work functions calculated for $\mathrm{Li}$ $(3.33 \mathrm{eV})$ and $\mathrm{Mg}(3.86 \mathrm{eV}) .{ }^{31}$ Hence, if we compare the left-hand panels of Figs. 6 and 7 we find a one-to-one correspondance between the amount of charge transfer at the surface and the resultant dipole barrier for these elements.

In the right-hand panel of Fig.7 we present an expanded view of the calculated envelope potentials for $\mathrm{Li}$, $\mathrm{Be}$, and $\mathrm{Mg}$ which clearly exhibit the same qualitative differences between the metals as seen in Fig. 6, i.e., the potential oscillates in $\mathrm{Li}$ and $\mathrm{Mg}$ while it decreases monotonically into the bulk in Be. In fact, this behavior is already apparent in jellium calculations of Lang and $\mathrm{Kohn}^{59}$ where one may observe that an $r_{s}$ value of approximately 2 separates a regime of high density in which the effective potential varies monotonically from a regime of low density in which the potential oscillates with an amplitude which increases with the $r_{s}$ value. In the jellium calculations, one may furthermore see that the effective potential at the position of the atom in the surface layer $(S)$ is below its bulk value only for densities corresponding to $r_{s}>2$. Now, the $r_{s}$ values of $\mathrm{Be}, \mathrm{Mg}$, and $\mathrm{Li}$ are $1.87,2.66$, and 3.25 , respectively, which immediately explains the signs and the relative magnitude of the surface layer potentials shown in Fig. 7.

If one compares the surface envelope potentials in the right-hand panel of Fig.7 with the calculated surface and subsurface SCLS for Be, Mg, and Li in Figs. 1 and 4 one finds a clear correlation which includes the anomalous sign in $\mathrm{Be}$, the monotonic and slow decrease in $\mathrm{Be}$ as well as the relative magnitudes of the shift in the sequence $\mathrm{Be}$, $\mathrm{Mg}$, and $\mathrm{Li}$. This close correlation may be written in the form

$$
\Delta_{c}(R) \sim-\Delta \bar{V}_{C}(R),
$$

suggested by the so-called initial-state picture. We shall return to this in connection with the discussion of the physical concept of complete screening in the following sections.

The decomposition in Fig. 7 of the subsurface potentials for beryllium shows that the dipole part plays a crucial role in all the layers, and becomes the dominant contribution in the second $(S-2)$ and third $(S-3)$ subsurface layers. It follows from direct examination of the $M^{s z}$ matrix that this potential is almost solely due to the existence of nonvanishing, unidirected dipoles in the $(S-2)-(S-5)$ layers as shown in Fig. 6. In addition, the effect of the dipoles is enhanced in Be due to its exceptionally small atomic volume and nearest-neighbor distance, so that the effective strength scaled by $M^{s z}$ is approximately $40 \%$ larger for Be than for $\mathrm{Mg}$.

The beryllium subsurface dipoles originate from the valence electron distribution, and it was pointed out in Ref. 28 that changes in the immediate surroundings of a Be bulk atom, such as the presence of a surface, have a comparatively large effect on the valence $2 p$ charge, owing to the fact that there is no $p$ core against which the $2 p$ orbitals would have to orthogonalize. This simple physical explanation of the behavior of beryllium SCLS appears to be consistent with the results of the present analysis, where we have probed singular characteristics in the surface electronic structure present already in the initial state, i.e., without a separate consideration of the electrostatics occurring in the final state.

\section{Band-center shifts}

The shift in the potential at a surface is directly responsible for a similar shift in the position of the surface $s$ and $p$ bands. This is shown in Fig. 8, where calculated band center surface shifts for the alkali and alkaline-earth metals are compared with the SCLS from the full impurity calculations. In the comparison, the band center is taken to be the second-order LMTO band-center parameter $C$ (Refs. 43 and 30 ) projected on the impurity atom and we include shifts referring to both the initial (perfect bulksurface) and final (relaxed bulk-surface impurity) states of the screening process. For simplicity and because they differ by less than $0.03 \mathrm{eV}$ only the mean value of the separate $s$ and $p$ band-center shifts is given. The variation of the SCLS through the two series of simple metals is seen to be exceedingly well reproduced by the surface band-center shifts, in complete analogy with the potential shifts observed above for the more restricted set of elements $\mathrm{Be}, \mathrm{Mg}$, and $\mathrm{Li}$. This close correlation also includes the reduction in SCLS between $\mathrm{Li}$ and $\mathrm{Na}$, the sign change between $\mathrm{Be}$ and $\mathrm{Mg}$, as well as most of the detailed features of the $\mathrm{Na}-\mathrm{Cs}$ and $\mathrm{Mg}$ - $\mathrm{Ba}$ sequences.

The observation that surface potential and band-center

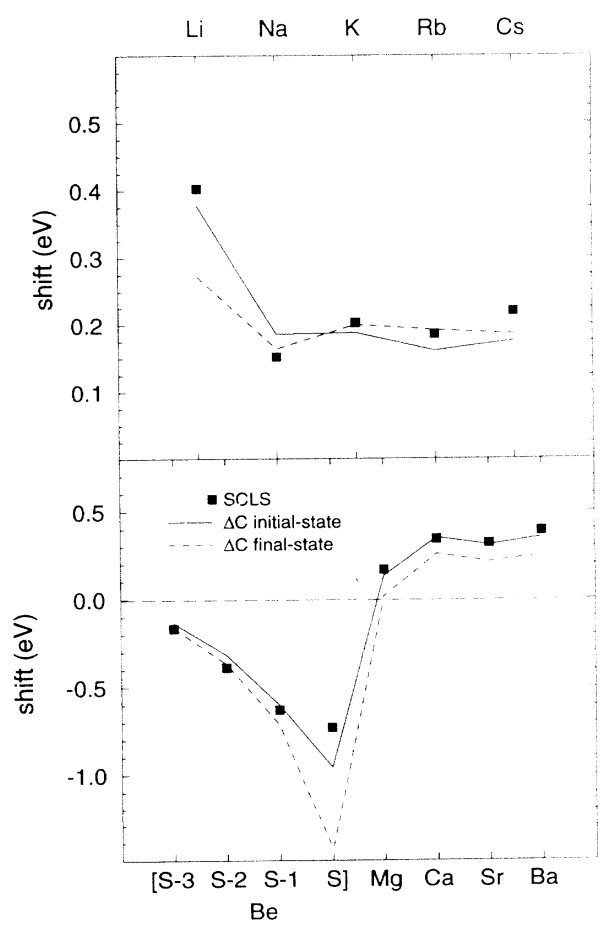

FIG. 8. Comparison between the surface shifts of the (average) $s p$ band centroids $(\Delta C)$ with the full SCLS calculations, for the alkali (top) and alkaline-earth (bottom) metals. The band-center shift is given for both the initial state (line) and impurity final state (dashed line) of the core-ionization process. Included to the left in the bottom panel are the layer-resolved shifts for $\mathrm{Be}$, where $S-1$ denotes the first subsurface layer, $S-2$ the second subsurface layer, etc. 
shifts resemble the total-energy results to such an extent that the impurity calculations to some extent become almost unnecessary, speaks in favor of the so-called initialstate effects for the SCLS of the simple metals. This is so because of the band centers and presumably also the core levels, on which the potential act appears to have accomplished the major part of the shift already in the initial state. This is consistent with earlier reports for the $\mathrm{Al}(100)$ surface, where Feibelman's theoretical "finalstate" shift of $-0.097 \mathrm{eV},{ }^{38}$ obtained by slab $(Z+1)$ impurity calculations, was found to be in good agreement with an initial-state ( $1 s$-eigenvalue) shift of $-0.120 \mathrm{eV},{ }^{57}$ whereby it was concluded that so-called final-state effects had only a minor influence on the aluminium SCLS. ${ }^{38}$ Hence, the SCLS of the simple metals appear to be particularly well described by the initial-state model. This is in contrast to the transition metals where the initialstate shifts overestimate the observed shifts by $\sim 0.2-0.3$ eV (Refs. 18, 10, and 11) and where the curves describing the initial-state and "true" values through a transition metal series are approximately translated by half an element. ${ }^{18,61,11}$

The initial-state potential shifts for the transition metals are explained in the literature ${ }^{12}$ as electrostatically induced due to the narrowing of the surface $d$ state density in conjunction with a preservation of the $d$ occupancy in the surface layer. This causes, e.g., opposite shifts for elements in the beginning and in the end, respectively, of a transition metal series. ${ }^{12,10}$ As pointed out by Riffe et al. ${ }^{15}$ such a simple model is not as readily applicable to the simple metals since they have free-electron-like conduction bands. Hence, quantitative understanding of the transfer of the $s p$ electrons in the surface region, which governs the potential shifts, seems to be difficult to obtain within, e.g., a tight-binding picture. On the other hand, the actual role played by the potential and band-center shifts is not self-evident within the the complete screening picture, since here the SCLS is entirely described in terms of final-state total energy differences. The agreement between the band-center shifts and the full SCLS is, however, too good to be overlooked, and what is needed therefore is a physical picture, within that of complete screening rather than Koopman's theorem, of the connection between these two quantities in order to assess the relevance of the potential shifts. That is, we would like to relate the surface segregation energy of the coreionized atom to the shift in the surface potential.

\section{One-electron contribution in sp-bonded metals}

The well-known Friedel ${ }^{62,63}$ type model for cohesion in the $d$-transition series is based on the notion that the ground state energetics, as characterized through a series by quantities such as the cohesive energy, are largely determined by the bonding properties of the $d$ electrons. This $d$ bonding is well described by the one-electron energies if these are grouped into bonding and antibonding states, respectively, separated naturally by the band centroid. Hence, for a transition metal the energy of transformation between (1) and (2) may be written ${ }^{62}$

$$
\begin{aligned}
\Delta E_{(2)-(1)}= & \int_{F}^{\epsilon_{F}^{2}}\left(\varepsilon-C_{d}^{(2)}\right) D_{d}^{(2)}(\varepsilon) d \varepsilon \\
& -\int^{\epsilon_{F}^{1}}\left(\varepsilon-C_{d}^{(1)}\right) D_{d}^{(1)}(\varepsilon) d \varepsilon
\end{aligned}
$$

in terms of the self-consistent state density $D_{d}$ and bandcenter $C_{d}$ of the $d$ electrons in the two different environments. Gelatt et $a l .^{64}$ have analyzed the various contributions to the cohesive energy of metals within renormalized-atom theory and finds that owing to substantial cancellations (9) provides a good representation of the energetics of transition metals.

If one assumes that the bonding in the $2 p$ and $3 p$ series is formed by an $s p$ hybrid with band-center $\hat{C}_{s p}$ the oneelecton contribution to the energy of a system with $n s p$ electrons may be written in the form

$$
E_{s p}=\int^{\epsilon_{F}}\left(\varepsilon-\hat{C}_{s p}^{n}\right) D_{s p}^{n}(\varepsilon) d \varepsilon
$$

In a core-ionization process (10) may describe the bonding in the initial state whereas the bonding in the final state may be given by a similar expression with $n+\delta n$ electrons and a shifted state density. Assuming that the local band shift between the initial and the final state may may be obtained in a rigid-band model, the initialand final-state bands are related by

$$
\begin{gathered}
D_{s p}^{n+\delta n}(\varepsilon) \cong D_{s p}^{n}(\varepsilon+\delta w), \\
\hat{C}_{s p}^{n+\delta n} \cong \hat{C}_{s p}^{n}-\delta w,
\end{gathered}
$$

where $\delta w$ is the energy by which the final-state levels are pulled down. Neglecting the impurity aspect, the $s p$ screening energy may be written

$$
\begin{aligned}
\Delta E_{s p}= & \int^{\epsilon_{F}}\left(\varepsilon-\hat{C}_{s p}^{n}\right) D_{s p}^{n}(\varepsilon) d \varepsilon \\
& -\int^{\epsilon_{F}}\left(\varepsilon-\hat{C}_{s p}^{n+\delta n}\right) D_{s p}^{n+\delta n}(\varepsilon) d \varepsilon,
\end{aligned}
$$

which, by the introduction of the "transition-state" center

$$
\hat{C}_{s p}^{n+\delta n / 2} \cong \hat{C}_{s p}^{n}-\delta w / 2 \cong \hat{C}_{s p}^{n+\delta n}+\delta w / 2
$$

becomes $^{11}$

$$
\Delta E_{s p} \approx\left(\epsilon_{F}-\hat{C}_{s p}^{n+\delta n / 2}\right) \delta n .
$$

In the core-ionization process $\delta n \cong 1$ and since the analysis applies equally well to bulk $(B)$ and surface $(S)$ atoms the SCLS may be related to the shift of $\hat{C}_{s p}^{n+\delta n / 2}$ between the bulk and surface, i.e.,

$$
\Delta_{c} \approx-\left(\hat{C}_{s p}^{S ; n+\delta n / 2}-\hat{C}_{s p}^{B ; n+\delta n / 2}\right)
$$

Hence, by considering the one-electron bonding energy of the $s p$ electrons we have arrived at a result which is consistent with the correlation between the SCLS and the band-center shifts observed in Fig. 8, and which justifies this behavior within the concept of complete screening. The small but systematic discrepancy between the SCLS and the "mean" (transition-state) value of the initial- and final-state band-center shift, may even be attributed to the neglect of the impurity effects in (16). 


\section{METALLIC ENERGETICS OF THE $2 P$ AND $3 P$ SERIES}

To help understand the behavior of the SCLS of the metallic elements in the second and third rows of the Periodic Table we shall present the atomic volume, the surface energy, and the SCLS of all the elements in the two series under the prerequisite that all the elements are treated as metals. The underlying calculations are performed under the constraint that the 4A-7A elements of the $2 p$ and $3 p$ series form in the fcc crystal structure and at the theoretically determined atomic volumes. Under these conditions the $p$-transition elements, which otherwise are insulating or semiconducting and order in complicated crystal structures, will exhibit metallic behavior. It should of course be stressed that because of these assumptions the following SCLS data may not be compared directly with experimentally observed values and with more realistic theoretical investigations. ${ }^{65}$ In the case of the surface energy, the present ASA results are nonetheless in reasonable agreement with the semiempirical values given by de Boer et al. ${ }^{66}$

In Fig. 9 we show the calculated equilibrium atomic volumes of the $2 p$ and $3 p$ series of elements obtained by the tight-binding LMTO method with an $s p d$ basis. The variation in atomic volume observed in the figure is the result of an almost linear contraction of the core size caused by incomplete screening of the increasing nuclear charge. To this is added a parabolic variation caused by a gradual increase in cohesion as the bonding states of a common $s p$ band are being filled followed by a gradual reduction when the antibonding states start to be occupied. The atomic volume across the $3 p$ series clearly exhibits the expected asymmetric parabolic variation with atomic number in complete analogy with the well-known behavior of the atomic volume in the $d$ transition series. In the $2 p$ series the core size is very small and rapidly contracting because there are no inner $p$ states which the $2 p$

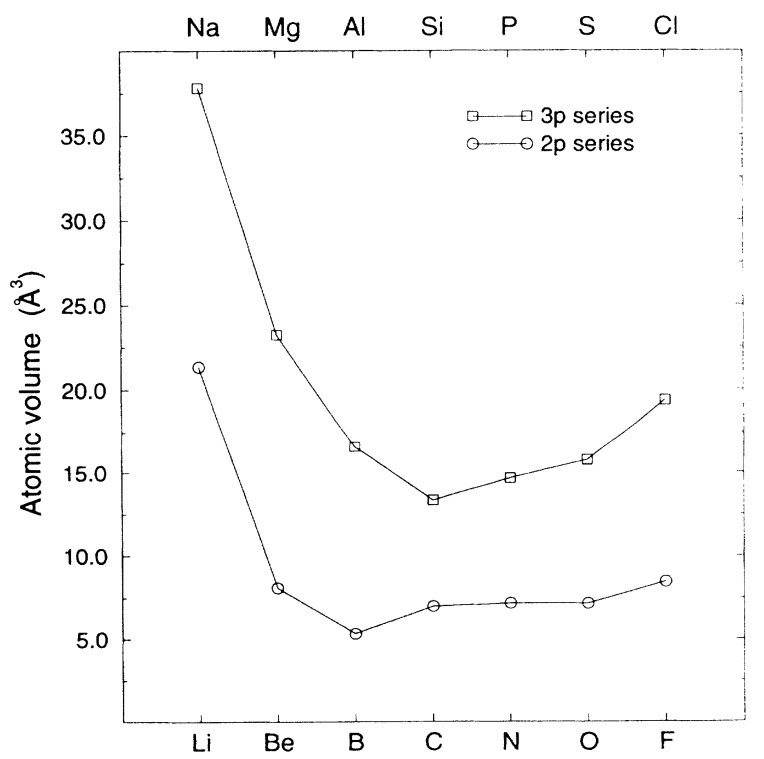

FIG. 9. Calculated equilibrium fcc-bulk atomic volumes, in units of $\AA^{3}$, for the $3 p$ series (top curve) and the $2 p$ series (bottom curve), respectively. states have to be orthogonal to. As a result, the atomic volumes of the $2 p$ series are much smaller than those of the $3 p$ series and the variation with atomic number is extremely asymmetric.

The calculated surface energy and surface core-level shift of the fcc(111) surface of the elements in the $2 p$ and $3 p$ series at their computed equilibrium volumes are presented in Figs. 10 and 11 . For the $3 p$ metals in Fig. 10 the important feature is the nearly parabolic variation of the surface energy with the filling of the common $s p$ band. This parabolic variation in conjunction with the simple estimate (2) immediately leads to the linear variation with atomic number as well as the sign change at the middle of the series in the SCLS shown in the lower panel of Fig. 10. The fact that $\mathrm{Al}$ is observed to have a zero or slightly negative shift, depending on the crystal face, may thus be directly interpreted as a "halffilling" effect in complete analogy with the case of, e.g., the $5 d$ transition metals, and gives perhaps the strongest support to the common $s p$ bonding versus anti-bonding interpretation as formulated by Eq. (13).

The surface energies calculated for the $2 p$ elements are shown in Fig. 11. Here, the variation with atomic number is more complicated than in the $3 p$ series and essentially reflects the less regular variation of the atomic volume. If one disregards the large surface energy of $\mathrm{Be}$ which is obviously caused by the strong bonding of the $2 s$ orbital and corresponding low atomic volume one may, however, observe a parabolic variation in the series with a "maximum" at C, i.e., the element which is isovalent with $\mathrm{Si}$ in the $3 p$ series.

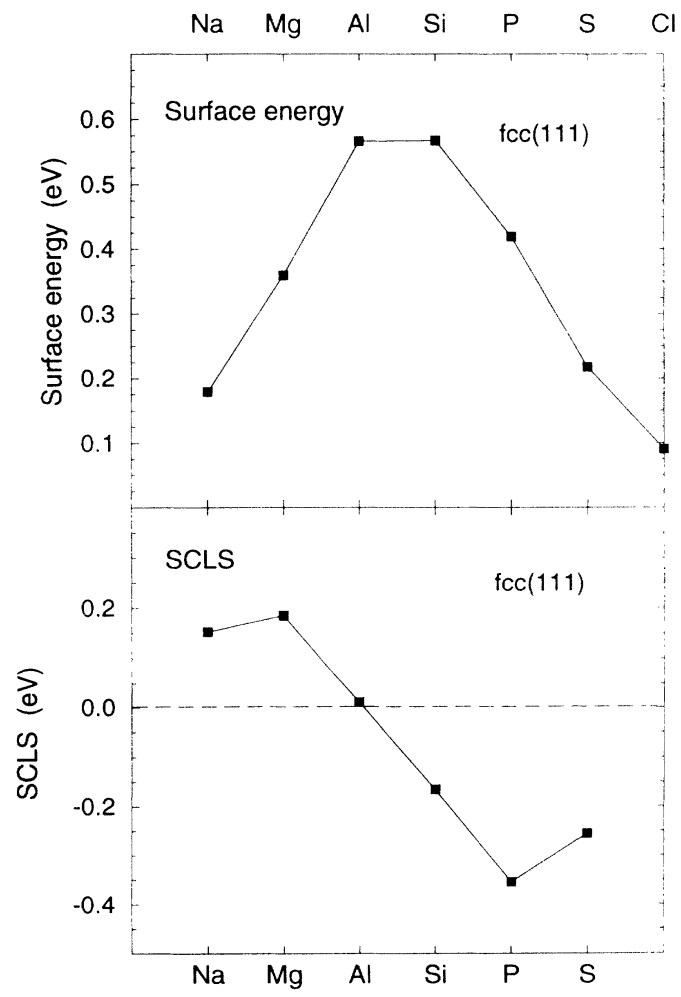

FIG. 10. Calculated surface energy (top) and $2 p$ surface core-level shift (bottom) for the fcc(111) surface of the $3 p$ series of elements. 


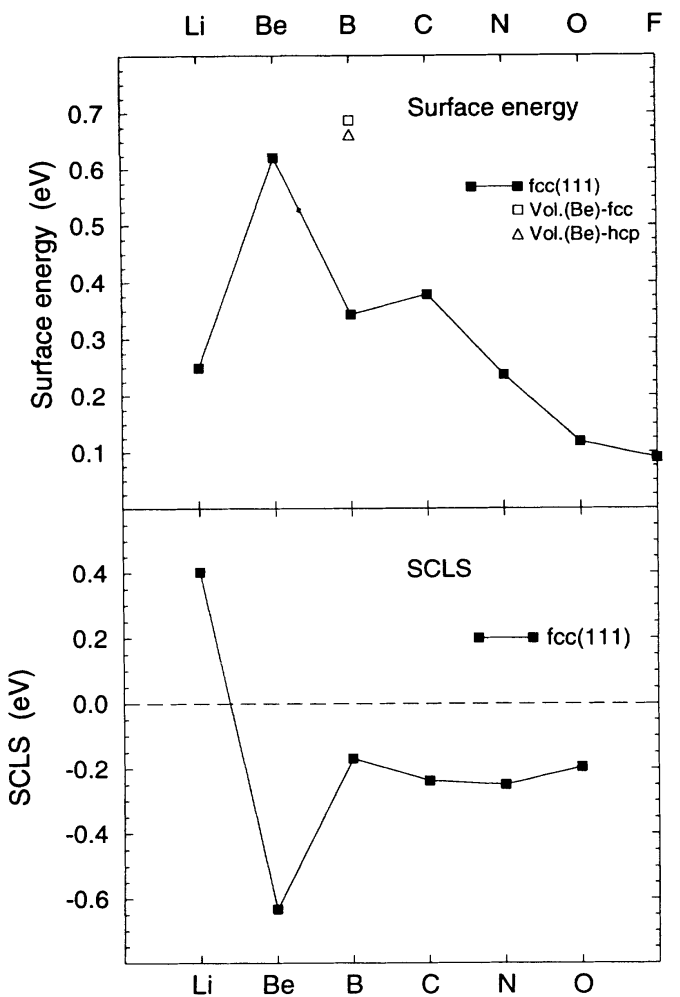

FIG. 11. Calculated surface energy (top) and $1 s$ surface core-level shift (bottom) for the fcc(111) surface of the $2 p$ series of elements. The two open symbols in the top panel refer to surface energy calculations for $B$ at the atomic volume of $\mathrm{Be}$, performed separately for both the fcc(111) and the hcp(0001) surface.

The calculated SCLS of the $2 p$ series in the lower panel of Fig. 11 are consistent with the behavior of the surface energies and the approximate relation (2) except for B where the $(\mathrm{Z}+1)$ approximation would predict a slightly positive SCLS. Hence, it is tempting to explain the large positive and negative shifts calculated for $\mathrm{Li}$ and $\mathrm{Be}$, respectively, completely in terms of the corresponding irregular variation of the surface energy in the sequence $\mathrm{Li}-\mathrm{Be}-\mathrm{B}$. However, if $\mathrm{B}$ is calculated at the atomic volume of $\mathrm{Be}$, as required in a consistent application of (2), we calculate the surface energy of $B$ to be slightly higher than that of Be. This result for B, included in Fig. 11, is in fact in better agreement with the surface energy reported by de Boer et al., using for the semiemperical estimate similar atomic volumes for $\mathrm{B}$ and $\mathrm{Be}{ }^{66}$ Hence, according to the data of de Boer et al. and to the present calculations with equal volumes for the $Z$ and $(Z+1)$ elements, the relation (2) yields an incorrect result and becomes of limited value for interpreting the Be shifts. One might then trivially argue that the "impurity contribution" is playing an important role for these shifts. It is clearly Be which deviates from a regular behavior in the metallic cohesive properties of the $2 p$ series, rather than the $(Z+1)$-element B.

\section{CONCLUSION}

We have calculated the surface core-level shifts for the most close-packed surfaces of the simple metals within the concept of complete screening which allows us to identify the SCLS with the surface segregation energy of a neutral, core-ionized atom. The surface calculations and the separate bulk-surface impurity calculations have been performed by means of Green's-function techniques within the tight-binding linear-muffin-tin-orbitals method, employing the frozen-core and atomic-sphere approximations except for the addition of a monopoledipole contribution to the electrostatic intersphere potential. In the case of the alkaline-earth metals, we have used an improved functional based on the complete valence charge density within overlapping atomic spheres. As a result, we obtain excellent agreement with the experimental SCLS data for all the simple metals studied with a maximum deviation of $0.1 \mathrm{eV}$ in $\mathrm{Li}, \mathrm{Be}$, and $\mathrm{Ba}$.

The origin of the simple metal SCLS has been analyzed in terms of layer-resolved surface shifts in the local potential, as caused by the separate contributions from the monopoles and dipoles in the surface region. For $\mathrm{Be}$, nonvanishing dipoles in the subsurface region cause an anomalously slow decay of the potential and core-level shifts as one proceeds into the bulk in agreement with experimental observations. We make the general observation that the SCLS, the potential shift, and band-center shift goes hand in hand in the simple metal surfaces. This correlation is interpreted within a simple one-electron screening model, using the notion of bonding and antibonding $s p$ states in analogy with the picture of $d$-electron bonding in the transition metals. We have thereby been able to conclude that the anomalous negative sign of the Be SCLS is an initial-state effect which is caused by the slow decay of the one-electron potential of a high-density electron gas already apparent in the onedimensional jellium description due to Lang and Kohn. ${ }^{59}$ Finally, we have performed surface energy and SCLS calculations for the (hypothetical) fcc(111) surface of the $2 p$ and $3 p$ series of elements. The zero SCLS exhibited by Al may thereby be explained as simply due to a half-filling effect occuring in the middle of the $3 s 3 p$ series.

\section{ACKNOWLEDGMENTS}

The Center for Atomic-scale Materials Physics is sponsored by the Danish National Research Foundation. Part of the work was supported by grants from the Danish research councils through the Danish Center for Surface Reactivity. B. Johansson is grateful for financial support from the Swedish Natural Science Research Council.
${ }^{1}$ P.H. Citrin, G.K. Wertheim, and Y. Baer, Phys. Rev. Lett. 41, 1425 (1978).

${ }^{2}$ G.K. Wertheim and G. Creselius, Phys. Rev. Lett. 40, 813 (1978).
${ }^{3}$ N. Mårtensson, H.B. Saalfeld, H. Kuhlenbeck, and M. Neumann, Phys. Rev. B 39, 8181 (1989).

${ }^{4}$ A, Flodström, R. Nyholm, and B. Johansson, in Advances in Surface and Interface Science, Volume 1: Techniques, 
edited by R.Z. Bachrach (Plenum Press, New York, 1992).

${ }^{5}$ M. Aldén, H.L. Skriver, and B. Johansson, Phys. Rev. Lett. 71, 2449 (1993).

${ }^{6}$ P.H. Citrin and G.K. Wertheim, Phys. Rev. B 27, 3176 (1983).

${ }^{7}$ D. Spanjaard, C. Guillot, M.C. Desjonquères, G. Tréglia, and J. Lecante, Surf. Sci. Rep. 5, 1 (1985).

${ }^{8}$ W.F. Egelhoff, Surf. Sci. Rep. 6, 253 (1987).

${ }^{9}$ A. Nilson, A. Stenborg, H. Tillborg, and N. Mårtensson, Phys. Rev. B 47, 13590 (1993).

${ }^{10}$ M. Methfessel, D. Hennig, and M. Scheffler, Surf. Sci. 287/288, 785 (1993).

${ }^{11}$ M. Aldén, I.A. Abrikosov, B. Johansson, N.M. Rosengaard, and H.L. Skriver, Phys. Rev. B 50, 5131 (1994).

${ }^{12}$ P.J. Feibelman and D.R. Hamann, Solid State Commun. 31, 413 (1979).

${ }^{13}$ G.K. Wertheim and D.N.E. Buchanan, Phys. Rev. B 43, 13815 (1991).

${ }^{14}$ D.M. Riffe, G.K. Wertheim, and P.H. Citrin, Phys. Rev. Lett 67, 116 (1991).

${ }^{15}$ D.M. Riffe, G.K. Wertheim, D.N.E. Buchanan, and P.H. Citrin, Phys. Rev. B 45, 6216 (1992).

${ }^{16}$ G.K. Wertheim, D.M. Riffe, and P.H. Citrin, Phys. Rev. B 45, 8703 (1992).

${ }^{17}$ B. Johansson and N. Mårtensson, Phys. Rev. B 21, 4427 (1980).

${ }^{18}$ B. Johansson and N. Mårtensson, Helv. Phys. Acta 56, 405 (1983).

${ }^{19}$ W. Eberhardt, G. Kalkoffen, and C. Kunz, Solid State Commun. 32, 901 (1979).

${ }^{20}$ T.-C. Chiang and D. Eastman, Phys. Rev. B 23, 6836 (1981)

${ }^{21}$ R. Kammerer, J. Barth, F. Gerken, and C. Kunz, S.A. Flodström, and L.I. Johansson, Phys. Rev. B 26, 3491 (1982).

${ }^{22}$ R. Nyholm, A.S. Flodström, L.I. Johansson, S.-E. Hörnström, and J. Schmidt-May, Surf. Sci. 149, 449 (1985).

${ }^{23}$ K. Jacobi, C. Astaldi, B. Frick, and P. Geng, Phys. Rev. B 36, 3079 (1987).

${ }^{24}$ P.S. Bagus, G. Pacchioni, and F. Parmigiani, Phys. Rev. B 43, 5172 (1991).

${ }^{25}$ R. Nyholm, J.N. Andersen, J.F. Van Acker, and M. Qvarford, Phys. Rev. B 44, 10987 (1992).

${ }^{26}$ E. Lundgren, J.N. Andersen, M. Qvarford, and R. Nyholm, Surf. Sci. 281, 83 (1993).

${ }^{27}$ L.I. Johansson, H.I.P. Johansson, J.N. Andersen, E. Lundgren, and R. Nyholm, Phys. Rev. Lett. 71, 2453 (1993).

${ }^{28}$ M. Aldén, H.L. Skriver, and B. Johansson, Phys. Rev. Lett. 71, 2457 (1993).

${ }^{29}$ Perhaps even more interesting spectral features have recently been observed for the hcp $(10 \overline{1} 0)$ face of beryllium, in H.I.P. Johansson, L.I. Johansson, E. Lundgren, J.N. Andersen, and R. Nyholm, Phys. Rev. B 49, 17460 (1994).

${ }^{30}$ H.L. Skriver and N.M. Rosengaard, Phys. Rev. B 43, 9538 (1991).

${ }^{31}$ H.L. Skriver and N.M. Rosengaard, Phys. Rev. B 46, 7157 (1992).

${ }^{32}$ H.L. Skriver and N.M. Rosengaard, Phys. Rev. B 45, 9410 (1992).

${ }^{33}$ M. Aldén, S. Mirbt, H.L. Skriver, N.M. Rosengaard, and B. Johansson, Phys. Rev. B 46, 6303 (1992).
${ }^{34}$ M. Aldén, H.L. Skriver, S. Mirbt, and B. Johansson, Phys. Rev. Lett. 69, 2296 (1992); Surf. Sci. 315, 157 (1994).

${ }^{35}$ S. Mirbt, H.L. Skriver, M. Aldén, and B. Johansson, Solid State Commun. 88, 331 (1993).

${ }^{36}$ N.M. Rosengaard and H.L. Skriver, Phys. Rev. B 47, 12 865 (1993).

${ }^{37}$ O. Gunnarsson, O. Jepsen, and O.K. Andersen, Phys. Rev. B 27, 7144 (1983).

${ }^{38}$ P.J. Feibelman, Phys. Rev. B 39, 4866 (1989).

${ }^{39}$ A. Rosengren and B. Johansson, Phys. Rev. B 23, 3852 (1981).

${ }^{40}$ W.F. Egelhoff, Phys. Rev. Lett. 50, 587 (1983).

${ }^{41}$ O.K. Andersen and O. Jepsen, Phys. Rev. Lett. 53, 2571 (1984)

42 O.K. Andersen, O. Jepsen, and D. Glötzel, in Highlights of Condensed-Matter Theory, edited by F. Bassani, F. Fumi, and M.P. Tosi (North Holland, New York, 1985).

${ }^{43}$ O.K. Andersen, Z. Pawlowska, and O. Jepsen, Phys. Rev. B 34, 5253 (1986)

${ }^{44}$ W.R.L. Lambrecht and O.K. Andersen, Surf. Sci. 178, 256 (1986).

${ }^{45}$ B. Velickỳ and J Kurdnovskỳ, Surf. Sci. 64, 411 (1977)

${ }^{46}$ B. Wenzien, J. Kudrnovskỳ, V. Drachal, and M. Sob, J. Phys. Condens. Matter 1, 9893 (1989).

${ }^{47}$ For the purpose of clarity the term $E_{S, Q_{s}}$ was not explicitly mentioned in Refs. 5 and 28 but included in the solution energies which therefore differ from those given in Ref. 11 and used in the present work.

48 J. Kollár, L. Vitos, and H.L. Skriver, Phys. Rev. B 49, 11288 (1994).

${ }^{49}$ L. Vitos, J. Kollár, and H.L. Skriver, Phys. Rev. B 49, 16694 (1994).

${ }^{50}$ S.H. Vosko, L. Wilk, and M. Nusair, Can. J. Phys. 58, 1200 (1980).

${ }^{51}$ D.G. Pettifor, CALPHAD 1, 305 (1977).

52 D.G. Pettifor, J. Phys. F 7, 613 (1977).

${ }^{53}$ D.G. Pettifor, J. Phys. F 8, 219 (1977).

${ }^{54}$ L.I. Johansson (private communication).

${ }^{55}$ H.L. Davis, J.B. Hannon, K.B. Ray, and E.W. Plummer, Phys. Rev. Lett. 68, 2632 (1992).

${ }^{56}$ P.J. Feibelman, Phys. Rev. B 46, 2532 (1992).

${ }^{57}$ E. Wimmer, M. Weinert, A.J. Freeman, and H. Krakauer, Phys. Rev. B 24, 2292 (1981).

${ }^{58}$ P.S. Bagus and G. Pacchioni, Phys. Rev. B 48, 15274 (1993).

${ }^{59}$ N. D. Lang, and W. Kohn, Phys. Rev. B 1, 4555 (1970).

${ }^{60}$ R. Monnier and J.P. Perdew, Phys. Rev. B 17, 2595 (1978).

${ }^{61}$ M. Said, M.C. Desjonquères, and D. Spanjaard, Phys. Rev. B 47, 4722 (1993).

62 J. Friedel, in The Physics of Metals I, edited by J.M. Ziman (Cambridge University Press, Cambridge, 1969).

${ }^{63}$ J. Friedel, Ann. Phys. (Paris) 1, 257 (1976).

${ }^{64}$ C.D. Gelatt, H. Ehrenreich, and R.E. Watson, Phys. Rev. B 15, 1613 (1977).

${ }^{65}$ E. Pehlke and M. Scheffler, Phys. Rev. Lett. 71, 2338 (1993).

${ }^{66}$ F.R. de Boer, R. Boom, W.C.M. Mattens, A.R. Miedema, and A.K. Niessen, in Cohesion in Metals, edited by F.R. de Boer and D.G. Pettifor (North-Holland, Amsterdam, 1988), Vol. I, p. 676. 